PROCEEDINGS OF THE

AMERICAN MATHEMATICAL SOCIETY

Volume 140, Number 12, December 2012, Pages 4053-4062

S 0002-9939(2012)11277-8

Article electronically published on April 3, 2012

\title{
A NOTE ON AUTOMORPHISMS AND BIRATIONAL TRANSFORMATIONS OF HOLOMORPHIC SYMPLECTIC MANIFOLDS
}

\author{
SAMUEL BOISSIÈRE AND ALESSANDRA SARTI
}

(Communicated by Lev Borisov)

\begin{abstract}
We give a necessary and sufficient condition for an automorphism of the Hilbert scheme of points on a K3 surface (not necessarily algebraic) to be induced by an automorphism of the surface. We prove furthermore that the group of birational transformations of a projective irreducible holomorphic symplectic manifold is finitely generated.
\end{abstract}

\section{INTRODUCTION}

Compact irreducible holomorphic symplectic manifolds are higher dimensional analogues of K3 surfaces and share many of their well-known properties. Their second integral cohomology space carries a natural weight-two Hodge structure and, due to a result of Beauville [5], it can be endowed with a natural nondegenerate quadratic form generalizing the intersection pairing of a K3 surface.

If $X$ is a compact irreducible holomorphic symplectic manifold, the group $\operatorname{Aut}(X)$ of its biholomorphic automorphisms is discrete, and any automorphism $f \in \operatorname{Aut}(X)$ induces by pull-pack an isometry of $H^{2}(X, \mathbb{Z})$ for the quadratic form $q_{X}$, yielding a natural map:

$$
\Phi: \operatorname{Aut}(X) \longrightarrow \mathrm{O}\left(H^{2}(X, \mathbb{Z}), q_{X}\right), f \mapsto f^{*} .
$$

It is an important issue to understand to what extent an automorphism of $X$ is determined by the isometry induced on $H^{2}(X, \mathbb{Z})$.

If $X$ is a K3 surface, the strong Torelli theorem of Burns-Rapoport 11] gives a precise answer: the map $\Phi$ is injective and for every isometry $\phi \in \mathrm{O}\left(H^{2}(X, \mathbb{Z}), q_{X}\right)$, which is an isomorphism of integral Hodge structures and maps a Kähler class of $X$ to a Kähler class, there exists an automorphism $f \in \operatorname{Aut}(X)$ such that $f^{*}=\phi$. Using this theorem and lattice theory results, Nikulin 25 obtained an essentially complete understanding of the finite abelian automorphism groups of K3 surfaces. These results have been extended to nonabelian groups by Mukai 23] and to infinite order automorphisms by McMullen 22. In higher dimensions, Debarre 14 and Namikawa 24] provided counterexamples to various analogues of the global Torelli theorem. However, Verbitsky [33] and Markman 20] recently proved the following version of the Global Torelli theorem. Denote by $\operatorname{Mon}^{2}(X) \subset \operatorname{GL}\left(H^{2}(X, \mathbb{Z})\right)$ the group of monodromy operators. The following version of the global Torelli theorem

Received by the editors September 14, 2010 and, in revised form, March 22, 2011 and May 18, 2011

2010 Mathematics Subject Classification. Primary 14C05.

Key words and phrases. Hilbert scheme, automorphisms, holomorphic symplectic varieties.

(C)2012 American Mathematical Society

Reverts to public domain 28 years from publication 
holds: for every $\phi \in \operatorname{Mon}^{2}(X)$ which is an isomorphism of integral Hodge structures and maps a Kähler class of $X$ to a Kähler class, there exists an automorphism $f \in \operatorname{Aut}(X)$ such that $f^{*}=\phi$.

It is difficult to construct interesting automorphisms of irreducible holomorphic symplectic manifolds. We refer to Beauville [3, 4, Boissière-Nieper-WißkirchenSarti 8], Camere [12, O'Grady [26] and Oguiso-Schröer [31] for some constructions concerning Hilbert schemes of two points on K3 surfaces, generalized Kummer varieties, Fano varieties of lines on cubic fourfolds, double covers of EPW sextics or O'Grady's resolutions of some moduli spaces of sheaves on K3 or abelian surfaces. The situation is particularly interesting when $X$ is the Hilbert scheme $S^{[n]}$ of $n$ points on a K3 surface $S$. In this case, Beauville [4, Proposition 10] proved that the map $\Phi$ is injective, and Markman [21] obtained a very precise characterization of the group of monodromy operators that could, in the near future, help in constructing new automorphisms. There is for the moment one standard way to construct automorphisms of $S^{[n]}$ by starting from automorphisms of $S$, yielding a morphism $\operatorname{Aut}(S) \rightarrow \operatorname{Aut}\left(S^{[n]}\right)$ whose image consists of natural [7] automorphisms of $S^{[n]}$. The first main result of this paper (Theorem 1) is a characterization of the automorphisms obtained this way. It is easy to see that all these automorphisms leave invariant the class in $H^{2}\left(S^{[n]}, \mathbb{Z}\right)$ of the exceptional divisor of $S^{[n]}$, so it is natural to ask whether this condition is sufficient: we give in Theorem 1 a positive answer. The proof given here relies on some relations between the Kähler cones of $S$ and $S^{[n]}$ stated in Lemma 1. Note that a precise conjecture for the determination of the Kähler cone of $S^{[n]}$ is provided by Hassett-Tschinkel [17, Conjecture 1.2].

Denote by $\operatorname{Bir}(X)$ the group of bimeromorphic transformations of an irreducible holomorphic symplectic manifold $X$. If $X$ is nonprojective, Oguiso [29, Theorem 1.5], [27, Theorem 1.4], [28, Theorem 1.6] proved that the groups $\operatorname{Aut}(X)$ and $\operatorname{Bir}(X)$ are finitely generated by showing that they are almost abelian of finite rank (meaning that they are isomorphic to some $\mathbb{Z}^{r}$, up to finite kernel and cokernel) and constructed projective examples where both groups are not almost abelian of finite rank. The second main result of this paper (Theorem 2) answers a question of Oguiso 27] by showing that when $X$ is projective, the group $\operatorname{Bir}(X)$ is finitely generated. The proof relies on the recent proof of the global Torelli theorem by Verbitsky and Markman [20, 33.

\section{BASIC TOOLS ON HOLOMORPHIC SYMPLECTIC MANIFOLDS}

Let $X$ be an irreducible holomorphic symplectic manifold of dimension $2 n$ (with $n \geq 1)$. In the Hodge decomposition $H^{2}(X, \mathbb{C})=H^{2,0}(X) \oplus H^{1,1}(X) \oplus H^{0,2}(X)$ we put $H^{1,1}(X)_{\mathbb{R}}:=H^{1,1}(X) \cap H^{2}(X, \mathbb{R})$. The Kähler cone $\mathcal{K}_{X}$ is the open convex cone in $H^{1,1}(X)_{\mathbb{R}}$ of classes which can be represented by a positive closed $(1,1)$-form.

We denote by $q_{X}$ the canonical Beauville-Bogomolov [5] symmetric bilinear form on $H^{2}(X, \mathbb{Z})$ (we keep this notation also for its extension to $H^{2}(X, \mathbb{R})$ and $H^{2}(X, \mathbb{C})$ and for the associated quadratic form). This form is nondegenerate of signature $\left(3, b_{2}(X)-3\right)$ on $H^{2}(X, \mathbb{R})$ and it is such that $H^{1,1}(X)$ is orthogonal to $H^{2,0}(X) \oplus H^{0,2}(X)$. The restriction of $q_{X}$ to $H^{1,1}(X)_{\mathbb{R}}$ has signature $\left(1, b_{2}(X)-3\right)$ and for every Kähler class $\omega \in \mathcal{K}_{X}$, we have $q_{X}(\omega)>0$.

Put $\mathcal{S}_{X}:=\left\{\alpha \in H^{1,1}(X)_{\mathbb{R}} \mid q_{X}(\alpha)>0\right\}$. The signature of $q_{X}$ implies that $\mathcal{S}_{X}$ is the disjoint union of two open convex cones: the one containing the Kähler cone is 
denoted by $\mathcal{C}_{X}$ and called the positive cone; the other component is denoted by $\mathcal{C}_{X}^{\prime}$ and we have the property: $x \in \mathcal{C}_{X} \Leftrightarrow(-x) \in \mathcal{C}_{X}^{\prime}$.

If $\omega \in \mathcal{K}_{X}$, then $q_{X}(\omega, \cdot)$ is strictly positive on $\mathcal{C}_{X}$ and for any effective divisor $D$ on $X$, we have $q_{X}(\omega,[D])>0[18, \S 1.11]$. In the particular case when $X$ is a K3 surface, this property characterizes the Kähler cone [2]: if $\omega \in \mathcal{C}_{X}$ is such that $q_{X}(\omega, d)>0$ for any class $d$ of an effective divisor on $X$ such that $d^{2}=-2$, then $\omega \in \mathcal{K}_{X}$

Denote by $\mathcal{A}_{X} \subset H^{1,1}(S)_{\mathbb{R}}$ the ample cone, generated by the first Chern classes $c_{1}(L)$ of the ample line bundles $L$ on $X$. We have $\mathcal{A}_{X} \subset \mathcal{K}_{X}$.

Let $\operatorname{Pef}_{\operatorname{tr}}(X) \subset H^{1,1}(X,)_{\mathbb{R}}$ be the set of pseudo-effective transcendental classes, i.e. classes which can be represented by a closed positive $(1,1)$-current. Results of Debarre [15, §3.3], Huybrechts [19, Proposition 1] and Boucksom [10] show that it is a convex closed cone such that $\mathcal{K}_{X} \subset \operatorname{Pef}_{\text {tr }}(X)$ and $\overline{\mathcal{C}_{X}} \subset \operatorname{Pef}_{\text {tr }}(X)$.

The Néron-Severi group is $\operatorname{NS}(X):=H^{1,1}(X)_{\mathbb{R}} \cap H^{2}(X, \mathbb{Z})$, of Picard number $\rho(X):=\operatorname{rk}(\mathrm{NS}(X))$, and the transcendental lattice $\mathrm{T}(X)$ is the orthogonal complement of $\operatorname{NS}(X)$ in $H^{2}(X, \mathbb{Z})$. We denote the signature of a lattice by $\left(n_{1}, n_{2}, n_{3}\right)$, where $n_{1}$ is the number of positive eigenvalues, $n_{2}$ the number of the zero eigenvalues and $n_{3}$ the number of the negative eigenvalues of the associated real quadratic form. There are three possibilities:

- hyperbolic type: $\mathrm{NS}(X)$ is nondegenerate, of signature $(1,0, \rho(X)-1)$ and $\mathrm{T}(X)$ has signature $\left(2,0, b_{2}(X)-\rho(X)-2\right)$;

- parabolic type: $\mathrm{NS}(X) \cap \mathrm{T}(X)$ is of dimension $1, \mathrm{NS}(X)$ has signature $(0,1, \rho(X)-1)$ and $\mathrm{T}(X)$ has signature $\left(2,1, b_{2}(X)-\rho(X)-3\right)$;

- elliptic type: $\operatorname{NS}(X)$ is negative definite, of signature $(0,0, \rho(X))$ and $\mathrm{T}(X)$ has signature $\left(3,0, b_{2}(X)-\rho(X)-3\right)$.

By Huybrechts [18, Theorem 3.11], $X$ is projective if and only if $\operatorname{NS}(X)$ is hyperbolic.

\section{The Hilbert scheme of points on a K3 surface}

Let $S$ be a K3 surface (not necessarily algebraic) and $n \geq 2$. We denote by $S^{n}$ the product of $n$ copies of $S, p_{i}: S^{n} \rightarrow S$ the projection onto the $i$-th factor, $S^{(n)}:=S^{n} / \mathfrak{S}_{n}$ the symmetric quotient of $S$, where the symmetric group $\mathfrak{S}_{n}$ acts by permutation of the variables, $\pi: S^{n} \rightarrow S^{(n)}$ the quotient map, $\Delta$ the union of all the diagonals of $S^{n}$ and $D:=\pi(\Delta)$ its image in $S^{(n)}$. We denote by $S^{[n]}$ the Hilbert scheme (or Douady space if $S$ is not algebraic) which parametrizes the analytic subspaces of $S$ of dimension zero and length $n$. By Beauville [5], $S^{[n]}$ is an irreducible holomorphic symplectic manifold. The Hilbert-Chow morphism (Douady-Barlet morphism in the nonalgebraic case) $\rho: S^{[n]} \rightarrow S^{(n)}$ is projective and bimeromorphic, and it is a resolution of singularities. We denote by $E:=\rho^{-1}(D)$ the exceptional divisor, which is irreducible.

There exists an injective morphism $\iota: H^{2}(S, \mathbb{C}) \rightarrow H^{2}\left(S^{[n]}, \mathbb{C}\right)$ such that

$$
H^{2}\left(S^{[n]}, \mathbb{C}\right)=\iota\left(H^{2}(S, \mathbb{C})\right) \oplus \mathbb{C}[E]
$$

(we set $e:=[E]$ ), which is constructed as follows: for $\alpha \in H^{2}(S, \mathbb{C})$, there exists a unique $\beta \in H^{2}\left(S^{(n)}, \mathbb{C}\right)$ such that $\pi^{*} \beta=p_{1}^{*} \alpha+\cdots+p_{n}^{*} \alpha$ and we put $\iota(\alpha):=\rho^{*} \beta$. The morphism $\iota$ is compatible with the Hodge decomposition [5, Proposition 6]. 
After normalisation, the form $q:=q_{S^{[n]}}$ satisfies $q(\iota(\alpha))=\alpha^{2}$ for $\alpha \in H^{2}(S, \mathbb{C})$, $q(e)=-8(n-1)$ and $e$ is orthogonal to $\iota\left(H^{2}(S, \mathbb{C})\right)$. There exists a class $\delta$ such that $2 \delta=e$ and $H^{2}\left(S^{[n]}, \mathbb{Z}\right)=\iota\left(H^{2}(S, \mathbb{Z})\right) \oplus \mathbb{Z} \delta$ (see [5]).

There exists a natural morphism of groups $-_{n}$ : $\operatorname{Pic}(S) \rightarrow \operatorname{Pic}\left(S^{[n]}\right)$ constructed as follows: for any line bundle $L \in \operatorname{Pic}(S)$, the line bundle $\bigotimes_{i=1}^{n} p_{i}^{*} L$ projects to a line bundle $\mathcal{L}$ on $\operatorname{Pic}\left(S^{(n)}\right)$ and one defines $L_{n}:=\rho^{*} \mathcal{L}$. By construction we have $c_{1}\left(L_{n}\right)=\iota\left(c_{1}(L)\right)$. Denoting by $(\operatorname{Pic}(S))_{n}$ the set of line bundles $L_{n}$, one has $\operatorname{Pic}\left(S^{[n]}\right) \cong(\operatorname{Pic}(S))_{n} \oplus \mathbb{Z D}$ with $\mathcal{D}^{2} \cong \mathcal{O}(-E)$ and $c_{1}(\mathcal{D})=-\delta$. The following lemma which compares the Kähler cones of $S$ and $S^{[n]}$ via $\iota$ is the key to Theorem 1 .

\section{Lemma 1.}

(1) $\iota\left(\mathcal{C}_{S}\right) \subset \mathcal{C}_{S[n]}$.

(2) If $\omega:=\iota\left(\omega_{0}\right)+\lambda e \in \mathcal{C}_{S^{[n]}}$, then $\omega_{0} \in \mathcal{C}_{S}$. We have

$$
\iota\left(\mathcal{C}_{S}\right)=\mathcal{C}_{S^{[n]}} \cap \iota\left(H^{1,1}(S)_{\mathbb{R}}\right) .
$$

(3) $\iota\left(\mathcal{K}_{S}\right) \cap \mathcal{K}_{S^{[n]}}=\emptyset$. If $\omega=\iota\left(\omega_{0}\right)+\lambda e \in \mathcal{K}_{S^{[n]}}$, then $\lambda<0$ and $\omega_{0} \in \mathcal{K}_{S}$.

Proof.

1. Let $Z_{n}$ be the isospectral Hilbert scheme, which is defined by Haiman [16] as the reduced fiber product of $S^{[n]}$ with $S^{n}$ over $S^{(n)}$ and $\widetilde{Z_{n}} \rightarrow Z_{n}$ a resolution of singularities. We have a commutative diagram

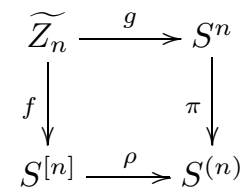

where $f$ and $g$ are surjective. For each $\alpha \in H^{2}(S, \mathbb{C})$, one sees immediately that $\iota(\alpha)$ is such that $f^{*} \iota(\alpha)=g^{*}\left(p_{1}^{*} \alpha+\cdots+p_{n}^{*} \alpha\right)$. For $\omega \in \mathcal{K}_{S}, \omega_{n}:=p_{1}^{*} \omega+\cdots+p_{n}^{*} \omega$ is a Kähler class of $S^{n}$; hence $g^{*}\left(\omega_{n}\right)$ is pseudo-effective, so $f^{*}(\iota(\omega)) \in \operatorname{Pef}_{\operatorname{tr}}\left(\widetilde{Z_{n}}\right)$. Since pseudo-effectivity is stable by considering the preimage (in the sense of currents, see Debarre [15, §3.1]) by surjective maps between compact varieties (a class is pseudoeffective if and only if its preimage is), this implies that $\iota(\omega) \in \operatorname{Pef}_{\mathrm{tr}}\left(S^{[n]}\right)$, which excludes that $\iota(\omega)$ is contained in the connected component $\mathcal{C}_{S^{[n]}}^{\prime}$. We conclude that $\iota\left(\mathcal{C}_{S}\right) \subset \mathcal{C}_{S[n]}$.

2. If $\omega=\iota\left(\omega_{0}\right)+\lambda e \in \mathcal{C}_{S^{[n]}}$, we have $\omega_{0}^{2}-8 \lambda^{2}(n-1)=q(\omega)>0$; hence $\omega_{0}^{2}>0$. Assume that $\omega_{0} \in \mathcal{C}_{S}^{\prime}$. Then $\left(-\omega_{0}\right) \in \mathcal{C}_{S}$, so by the first assertion and by convexity we get $\omega+\iota\left(-\omega_{0}\right)=\lambda e \in \mathcal{C}_{S^{[n]}}$, which is absurd; hence $\omega_{0} \in \mathcal{C}_{S}$.

3. If $\omega \in \mathcal{K}_{S}$, then $q(\iota(\omega), e)=0$; hence $\iota(\omega)$ is not a Kähler class for $S^{[n]}$. If $\omega=\iota\left(\omega_{0}\right)+\lambda e \in \mathcal{K}_{S^{[n]}}$, we have $0<q(\omega, e)=-8 \lambda(n-1)$ since $e$ is the class of an effective divisor, which implies $\lambda<0$. By the second assertion we have $\omega_{0} \in \mathcal{C}_{S}$. If $\omega_{0} \notin \mathcal{K}_{S}$ there exists an effective divisor $D$ on $S$ such that $\omega_{0} \cdot[D] \leq 0$. Hence $\iota([D])$ is again the class of an effective divisor, and we have $q(\omega, \iota([D]))=\omega_{0} \cdot[D] \leq 0$, a contradiction.

Remark 1. The argument used for the first assertion has been communicated to us by Daniel Huybrechts. If $S$ is algebraic (hence projective), one can argue in a different way, avoiding the use of currents. Denote by $\Xi_{n} \subset S \times S^{[n]}$ the universal family and by $p, q$ the respective projections on $S$ and $S^{[n]}$; the morphism $p$ is flat. 
For $L \in \operatorname{Pic}(S)$, put $\psi(L):=\operatorname{det}\left(q_{*} p^{*} L\right) \in \operatorname{Pic}\left(S^{[n]}\right)$. Beltrametti-Sommese 6, Theorem A.1] proved that the map $\psi: \operatorname{Pic}(S) \rightarrow \operatorname{Pic}\left(S^{[n]}\right)$ satisfies

$$
\psi(L)=L_{n} \otimes \mathcal{O}(-E) .
$$

By Catanese-Göttsche [13], $\psi(L)$ is very ample if and only if $L$ is $n$-very ample, which means that for any zero dimensional subscheme $\xi \subset S$ of length less than or equal to $n$, the canonical map $H^{0}(S, L) \rightarrow H^{0}\left(S, L \otimes \mathcal{O}_{\xi}\right)$ is surjective. In particular, if $L$ is ample, then for $k$ big enough, $L^{k}$ is very ample and $L^{k n}$ is $n$-very ample [6, Lemma 0.1.1]; hence $\psi\left(L^{k n}\right)$ is very ample. Since $S$ is projective, there exists a very ample line bundle $L$; hence $c_{1}(L) \in \mathcal{A}_{S}$. We get

$$
c_{1}\left(\psi\left(L^{k n}\right)\right)=k n \cdot \iota\left(c_{1}(L)\right)-e \in \mathcal{A}_{S[n]},
$$

which excludes that $\iota\left(c_{1}(L)\right) \in \mathcal{C}_{S}^{\prime}{ }^{[n]}$; otherwise, by convexity, we would have $e \in \mathcal{C}_{S^{[n]}}^{\prime}$.

\section{Classification of natural automorphisms}

Let $S$ be a K3 surface and $n \geq 2$. Any automorphism $\psi \in \operatorname{Aut}(S)$ induces an automorphism denoted $\psi^{[n]} \in \operatorname{Aut}\left(S^{[n]}\right)$, called natural, and the induced morphism $\operatorname{Aut}(S) \rightarrow \operatorname{Aut}\left(S^{[n]}\right)$ is injective [7]. By the relation $\psi^{[n]} \circ \rho=\rho \circ \psi^{(n)}$, where $\psi^{(n)}$ is the automorphism of $S^{(n)}$ induced by $\psi$, and by the fact that a natural automorphism leaves globally invariant the exceptional divisor $E$, one obtains that the action of $\psi^{[n]}$ on $H^{2}\left(S^{[n]}, \mathbb{Z}\right)$ can be decomposed as $\left(\psi^{[n]}\right)^{*}=\left(\psi^{*}\right.$,id) in the decomposition $H^{2}\left(S^{[n]}, \mathbb{Z}\right) \cong H^{2}(S, \mathbb{Z}) \oplus \mathbb{Z} \delta$ (where $\iota$ is implicit).

Let $f \in \operatorname{Aut}\left(S^{[n]}\right)$. Since the exceptional divisor $E$ is rigid, the geometric property $f(E)=E$ (i.e. $E$ is globally invariant) is equivalent to the algebraic property $f^{*} e=e$. We prove that this only condition characterizes the natural automorphisms.

Theorem 1. Let $S$ be a K3 surface and $n \geq 2$. An automorphism $f$ of $S^{[n]}$ is natural if and only if it leaves globally invariant the exceptional divisor.

Proof. The automorphism $f$ induces an isometry $f^{*}$ of the lattice $\left(H^{2}\left(S^{[n]}, \mathbb{Z}\right), q\right)$, and if $f$ leaves globally invariant the exceptional divisor, we have $f^{*}(\delta)=\delta$. Since $\delta$ is orthogonal to $\iota\left(H^{2}(S, \mathbb{Z})\right)$ for the form $q, f^{*}$ stabilizes $\iota\left(H^{2}(S, \mathbb{Z})\right)$; hence it decomposes as $f^{*}=(\varphi$, id $)$, where $\varphi$ is a Hodge isometry of the lattice $H^{2}(S, \mathbb{Z})$ since $\iota$ is compatible with the Hodge decomposition, as explained in 92 .

Let $\omega \in \mathcal{K}_{S^{[n]}}$. By Lemma 1 one can decompose it as $\omega=\iota\left(\omega_{0}\right)+\lambda e$ with $\omega_{0} \in \mathcal{K}_{S}$. Then

$$
f^{*}(\omega)=\iota\left(\varphi\left(\omega_{0}\right)\right)+\lambda e \in \mathcal{K}_{S[n]},
$$

so by Lemma 1 again it follows that $\varphi\left(\omega_{0}\right) \in \mathcal{K}_{S}$. By the global Torelli theorem for K3 surfaces, the effective Hodge isometry $\varphi$ is induced by a unique automorphism $\psi$ of $S$ such that $\psi^{*}=\varphi$.

The natural automorphism $\psi^{[n]}$ of $S^{[n]}$ induced by $\psi$ satisfies $\left(\psi^{[n]}\right)^{*}(\delta)=\delta$ and $\left(\psi^{[n]}\right)_{\mid H^{2}(S, \mathbb{Z})}^{*}=\varphi$, so $\left(\psi^{[n]}\right)^{*}=f^{*}$ on $H^{2}\left(S^{[n]}, \mathbb{Z}\right)$. By Beauville [4, Proposition 10] the map $\operatorname{Aut}\left(S^{[n]}\right) \rightarrow \mathrm{O}\left(H^{2}\left(S^{[n]}, \mathbb{Z}\right)\right)$ is injective, so $f=\psi^{[n]}$.

Remark 2. A similar classification result of automorphisms of generalized Kummer varieties is proven by Boissière-Nieper-Wißkirchen-Sarti [8, Theorem 4.1]. The proof there uses different techniques since the generalized Kummer varieties admit 
nontrivial automorphisms which act trivially on the second cohomology group 8 Corollary 4.3].

\section{Applications and examples}

Let $S$ be a K3 surface and $n \geq 2$. For $f \in \operatorname{Aut}\left(S^{[n]}\right)$, we define the index of $f$ by

$$
\lambda(f):=\frac{q\left(f^{*}(e), e\right)}{q(e)},
$$

in such a way that in $\operatorname{NS}\left(S^{[n]}\right)$ we have $f^{*}(e)=\lambda(f) e+\iota(d)$ for some $d \in \operatorname{NS}(S)$. Any natural automorphism has index 1 , and for any natural automorphism $f$ and any automorphism $g \in \operatorname{Aut}\left(S^{[n]}\right)$ we have

$$
\lambda(f \circ g)=\lambda(g)=\lambda(g \circ f) .
$$

In particular $\lambda\left(f \circ g \circ f^{-1}\right)=\lambda(g)$; hence the index is invariant for the action of $\operatorname{Aut}(S)$ on $\operatorname{Aut}\left(S^{[n]}\right)$ by conjugation.

Boissière 17. Proposition 2] proves by topological arguments that if $\rho(S)=0$, then all automorphisms of $S^{[n]}$ are natural. Theorem 11 gives in particular an algebraic argument. We study here the case $\rho(S) \geq 1$.

Proposition 1. Let $S$ be a $K 3$ surface and $n \geq 2$. If $S$ is of elliptic type or of hyperbolic type with $\rho(S)=1$, then $f \in \operatorname{Aut}\left(S^{[n]}\right)$ is natural if and only if $\lambda(f)=1$.

Proof. If $\lambda(f)=1$, then $f^{*}(e)=e+\iota(d)$. Since $f^{*}$ is an isometry, we get $d^{2}=0$. If $S$ is of elliptic type, this implies that $d=0$; hence $f^{*}(e)=e$ and by Theorem 1 , $f$ is a natural automorphism. The other implication is clear. In the hyperbolic case with $\rho(S)=1$, one has $\operatorname{NS}(S) \cong \mathbb{Z} d$ with $d^{2}>0$ and the argument is similar.

It would be interesting to understand if the invariant $\lambda: \operatorname{Aut}\left(S^{[n]}\right) \rightarrow \mathbb{Z}$ is enough to characterize the natural automorphisms. The previous result shows that this is the case for the generic algebraic K3 surface and in the elliptic case. It is not easy to find examples of nonnatural automorphisms of $S^{[n]}$. In fact there are, up to now, only two known examples. Take $S \subset \mathbb{P}_{3}$ to be a generic K3 surface containing no line. Beauville [4, §6] constructs an involution $i$ on $S^{[2]}$ as follows: for a reduced subscheme $\xi \in S^{[2]}$, the line $L$ through the two points in the support of $\xi$ cuts the surface $S$ in another length two subscheme $\xi^{\prime} \in S^{[2]}$. One can prove that the obtained birational map $i: S^{[2]} \rightarrow S^{[2]}, \xi \mapsto \xi^{\prime}$ extends to an automorphism. Denoting by $h$ the class of a hyperplane divisor, one computes that $i^{*}(\delta)=-3 \delta+4 h$ and $i^{*}(h)=-4 \delta+3 h$ (see Debarre [14, Théorème 4.1] or Oguiso [30, Lemma 4.3]). Since $\lambda(i)=-3$, this involution is not natural. The second example, which is inspired by the first one, is due to Oguiso (30; ; see also Amerik [1]). Consider a K3 surface $S$ admitting two embeddings as a quartic in $\mathbb{P}_{3}$, given by two different very ample line bundles $H_{1}, H_{2}$, whose classes are denoted by $h_{1}, h_{2}$. Each embedding induces an involution $i_{1}, i_{2}$ on $S^{[2]}$ as before, satisfying the following relations:

$$
\begin{aligned}
i_{j}^{*} h_{j} & =3 h_{j}-4 \delta, & i_{j}^{*} \delta & =-3 \delta+2 h_{j}, \quad j=1,2, \\
i_{1}^{*} h_{2} & =8 h_{1}-h_{2}-8 \delta, & i_{2}^{*} h_{1} & =8 h_{2}-h_{1}-8 \delta .
\end{aligned}
$$

Consider the composition $i=i_{1} \circ i_{2}$. Then $i^{*} \delta=10 h_{1}-2 h_{2}-7 \delta$, so $\lambda(i)=-7$ and $i$ is not natural (see also Oguiso [30, Lemma 4.6]). One can easily see from these computations that $i$ is of infinite order. 


\section{THE GROUP OF BIRATIONAL TRANSFORMATIONS}

Let $X$ be a projective irreducible holomorphic symplectic manifold. Using the global Torelli theorem of Verbitsky [33] and Markman [20, we prove that the group $\operatorname{Bir}(X)$ is finitely generated. Our proof is a generalization of the argument of Sterk [32] related to the automorphism group of projective K3 surfaces.

Given $f \in \operatorname{Bir}(X)$, the correspondence by the closure in $X \times X$ of the graph of $f$ induces an automorphism $f^{*} \in \operatorname{GL}\left(H^{2}(X, \mathbb{Z})\right.$ ). It is known (see Markman 20 and the references therein) that $f^{*} \in \mathrm{O}\left(H^{2}(X, \mathbb{Z}), q_{X}\right)$ and $f^{*} \in \operatorname{Mon}_{\mathrm{Hdg}}^{2}(X)$, where $\operatorname{Mon}_{\mathrm{Hdg}}^{2}(X)$ denotes the subgroup of $\operatorname{Mon}^{2}(X)$ of monodromy operators preserving the Hodge structure. Consider the group of birational transformations preserving the holomorphic two-form:

$$
\operatorname{Bir}^{0}(X):=\left\{f \in \operatorname{Bir}(X) \mid f^{*} \omega_{X}=\omega_{X}\right\} .
$$

Since every $f \in \operatorname{Bir}(X)$ induces a Hodge isometry of $H^{2}(X, \mathbb{Z})$ and $H^{2,0}(X)$ is generated by $\omega_{X}$, there is a character $\chi: \operatorname{Bir}(X) \longrightarrow \mathbb{C}^{*}$ defined by $f^{*} \omega_{X}=\chi(f) \omega_{X}$ and $\operatorname{Bir}^{0}(X)=\operatorname{Ker}(\chi)$ is a normal subgroup of $\operatorname{Bir}(X)$.

Lemma 2. Let $X$ be a projective irreducible holomorphic symplectic manifold. The quotient $\operatorname{Bir}(X) / \operatorname{Bir}^{0}(X)$ is a finite cyclic group.

Proof. The argument is similar as in Sterk [32, Lemma 2.1] or Beauville [4, Proposition 7]. Set $\mathrm{T}(X)_{\mathbb{R}}:=\mathrm{T}(X) \otimes_{\mathbb{Z}} \mathbb{R}$ and $E:=\left(H^{2,0}(X) \oplus H^{0,2}(X)\right) \cap H^{2}(X, \mathbb{R})$. There is an orthogonal decomposition:

$$
\mathrm{T}(X)_{\mathbb{R}}=E \oplus\left(\mathrm{T}(X)_{\mathbb{R}} \cap H^{1,1}(X)\right) .
$$

Since $X$ is projective, $\mathrm{T}(X)$ is of signature $(2,0, \rho(X)-2)$. Note that $q_{X}$ is positive on $E$, so it is negative on the second space. The isometry $f^{*} \in \mathrm{O}(\mathrm{T}(X))$ induced by $f \in \operatorname{Bir}(X)$ preserves this decomposition and it is unitary on each space, so the eigenvalues of $f^{*} \in O\left(T(X) \otimes_{\mathbb{Z}} \mathbb{C}\right)$ have modulus 1 . Since they are algebraic integers, they are roots of unity. In particular, the minimal polynomial of $\chi(f)$ is a cyclotomic polynomial $\Phi_{n}$ for some integer $n$. The polynomial $\Phi_{n}$ divides the characteristic polynomial of $f^{*} \in \mathrm{GL}(\mathrm{T}(X))$, so the Euler number of $n$ is smaller than or equal to $\operatorname{rk}(\mathrm{T}(X))$ and the possible values for $n$ are bounded. This shows that $\chi(\operatorname{Bir}(X)) \subset \mathbb{C}^{*}$ is a finite group.

Theorem 2. If $X$ is a projective irreducible holomorphic symplectic manifold, then $\operatorname{Bir}(X)$ is a finitely generated group.

Proof. We follow Markman [20 to generalize Sterk's argument [32, Proposition 2.2]. By Lemma 2, it suffices to prove that $\operatorname{Bir}^{0}(X)$ is finitely generated. Consider the restriction morphism:

$$
\rho: \operatorname{Mon}_{\mathrm{Hdg}}^{2}(X) \longrightarrow \mathrm{O}(\mathrm{NS}(X)) .
$$

Set $\mathcal{C}_{\mathrm{NS}}:=\mathcal{C}_{X} \cap \mathrm{NS}(X)$ and $\mathrm{O}^{+}(\mathrm{NS}(X)):=\left\{g \in \mathrm{O}(\mathrm{NS}(X)) \mid g\left(\mathcal{C}_{\mathrm{NS}}\right)=\mathcal{C}_{\mathrm{NS}}\right\}$. By Markman [20, Lemma 6.23], the group $\Gamma:=\operatorname{Im}(\rho)$ is an arithmetic subgroup of finite index in $\mathrm{O}^{+}(\mathrm{NS}(X))$, so $\Gamma$ is finitely generated [9, Theorem 6.12]. Define

$$
\Gamma_{\mathrm{T}}:=\left\{g \in \Gamma \mid g_{\mid \mathrm{T}(X)}=\mathrm{id}\right\} .
$$

The group $\Gamma_{T}$ is an arithmetic subgroup of $\Gamma$, so it is again finitely generated. Denote by $\mathcal{P} e x$ the set of prime exceptional divisors of $X$ (i.e. the set of reduced irreducible effective divisors of $X$ whose Gram matrix is negative definite). Set 
$W_{\text {Exc }}$ to be the subgroup of $\operatorname{Mon}_{\mathrm{Hdg}}^{2}(X)$ generated by reflections by elements of $\mathcal{P}$ ex [20, Definition 6.8, Theorem 6.18]. Define

$$
\begin{aligned}
\Gamma_{\mathrm{Bir}} & :=\{g \in \Gamma \mid g(\mathcal{P} e x)=\mathcal{P} e x\}, \\
\Gamma_{\mathrm{T}, \mathrm{Bir}} & :=\left\{g \in \Gamma_{\mathrm{T}} \mid g(\mathcal{P} e x)=\mathcal{P} \text { ex }\right\} .
\end{aligned}
$$

One has a semi-direct decomposition $\Gamma \cong \rho\left(W_{\text {Exc }}\right) \rtimes \Gamma_{\text {Bir }}$ 20, Lemma 6.23]. Since elements of $W_{\text {Exc }}$ act trivially on $\mathrm{T}(X)$, one deduces that $\Gamma_{\mathrm{T}} \cong \rho\left(W_{\mathrm{Exc}}\right) \rtimes \Gamma_{\mathrm{T}, \mathrm{Bir}}$, so $\Gamma_{\mathrm{T}, \text { Bir }}$ is a quotient of $\Gamma_{\mathrm{T}}$; hence it is finitely generated.

Denoting by $\operatorname{Mon}_{\text {Bir }}^{2}(X)$ the subgroup of $\operatorname{Mon}_{\mathrm{Hdg}}^{2}(X)$ of monodromy operators induced by birational transformations of $X$, one has $\operatorname{Ker}(\rho) \subset \operatorname{Mon}_{\text {Bir }}^{2}(X)$ and $\Gamma_{\text {Bir }} \cong \operatorname{Mon}_{\text {Bir }}^{2}(X) / \operatorname{Ker}(\rho)$ (see [20, Lemma 6.23 and (3) p. 26]), so

$$
\begin{aligned}
\Gamma_{\mathrm{T}, \mathrm{Bir}} & \cong\left\{g \in \operatorname{Mon}_{\mathrm{Bir}}^{2}(X) \mid g_{\mid \mathrm{T}(X)}=\mathrm{id}\right\} /\left\{g \in \operatorname{Mon}_{\mathrm{Bir}}^{2}(X) \mid g_{\mid \mathrm{NS}(X)}=\mathrm{id}\right\} \\
& \cong\left\{f \in \operatorname{Bir}(X) \mid f_{\mid \mathrm{T}(X)}^{*}=\mathrm{id}\right\} /\left\{f \in \operatorname{Bir}(X) \mid f_{\mid H^{2}(X, \mathbb{Z})}^{*}=\mathrm{id}\right\} .
\end{aligned}
$$

Since $X$ is projective, one has $\operatorname{Bir}^{0}(X) \cong\left\{f \in \operatorname{Bir}(X) \mid f_{\mid \mathrm{T}(X)}^{*}=\mathrm{id}\right\}$, and it is well known that $\left\{f \in \operatorname{Bir}(X) \mid f_{\mid H^{2}(X, \mathbb{Z})}^{*}=\mathrm{id}\right\}$ is a finite group (see [18, Proposition 9.1]): a birational transformation acting trivially on the second cohomology space leaves invariant a Kähler class, so extends to an automorphism by Fujiki's theorem and is an isometry for the Calabi-Yau metric uniquely associated to this Kähler class; the assertion follows then from the fact that the group of isometries of a compact Riemannian manifold is compact and $\operatorname{Aut}(X)$ is discrete. As a consequence, $\operatorname{Bir}^{0}(X)$ is finitely generated.

As mentioned by Oguiso [27, Question 1.6], it is not known whether $\operatorname{Aut}(X)$ is of finite index in $\operatorname{Bir}(X)$, so the following question remains open:

Question 1. For $X$ a projective irreducible holomorphic symplectic manifold, is the group $\operatorname{Aut}(X)$ finitely generated?

The key difference between understanding automorphisms and birational transformations of $X$ via various types of subgroups of $\mathrm{O}(\mathrm{NS}(X))$ is that, in the moduli space of marked irreducible holomorphic symplectic manifolds, two elements with the same period are always bimeromorphic [18, Theorem 4.3] but, contrary to the case of K3 surfaces, in higher dimensions they are not automatically isomorphic. This also explains why constructing automorphisms of $X$ from the action on the lattice $H^{2}(X, \mathbb{Z})$ is much more difficult for higher dimensional irreducible holomorphic symplectic manifolds than for K3 surfaces.

\section{ACKNOWLEDGEMENTS}

The authors thank Daniel Huybrechts and Manfred Lehn for their remarks and the referee for helpful comments and references improving the paper.

\section{REFERENCES}

1. E. Amerik, On an automorphism of Hilb ${ }^{[2]}$ of certain K3 surfaces, Proc. Edinburgh Math. Soc. (2) 54 (2011), 1-7. MR22764400

2. W. Barth, C. Peters, and A. Van de Ven, Compact complex surfaces, Ergebnisse der Mathematik und ihrer Grenzgebiete (3) [Results in Mathematics and Related Areas (3)], vol. 4, Springer-Verlag, Berlin, 1984. MR749574 (86c:32026) 
3. A. Beauville, Antisymplectic involutions of holomorphic symplectic manifolds, J. of Topology 4, no. 2 (2011), 300-304. MR2805992

4. __ Some remarks on Kähler manifolds with $c_{1}=0$, Classification of algebraic and analytic manifolds, Proc. Symp., Katata/Jap., 1982, Prog. Math. 39, 1-26 (1983). MR728605 (86c:32031)

5. - Variétés Kähleriennes dont la première classe de Chern est nulle, J. Differential Geom. 18 (1983), no. 4, 755-782. MR730926 (86c:32030)

6. M. Beltrametti and A. J. Sommese, Zero cycles and kth order embeddings of smooth projective surfaces, Problems in the theory of surfaces and their classification (Cortona, 1988), Sympos. Math., XXXII, Academic Press, London, 1991, with an appendix by Lothar Göttsche, pp. 3348. MR1273371 (95d:14005)

7. S. Boissière, Automorphismes naturels de l'espace de Douady de points sur une surface, Canad. J. Math. (2009), to appear.

8. S. Boissière, M. Nieper-Wißkirchen, and A. Sarti, Higher dimensional Enriques varieties and automorphisms of generalized Kummer varieties, J. Math. Pures Appl. 95 (2011), 553-563. MR 2786223

9. Armand Borel and Harish-Chandra, Arithmetic subgroups of algebraic groups, Ann. of Math. (2) 75 (1962), 485-535. MR0147566 (26:5081)

10. S. Boucksom, Cônes positifs des variétés complexes compactes, Thèse, Grenoble, 2002.

11. D. Burns and M. Rapoport, On the Torelli problem for kählerian K3 surfaces, Ann. Sci. École Norm. Sup. (4) 8 (1975), no. 2, 235-273. MR0447635 (56:5945)

12. C. Camere, Symplectic involutions of holomorphic symplectic fourfolds, arXiv:1010.2607.

13. F. Catanese and L. Gœttsche, d-very-ample line bundles and embeddings of Hilbert schemes of 0-cycles, Manuscripta Math. 68 (1990), no. 3, 337-341. MR1065935 (91i:14012)

14. O. Debarre, Un contre-exemple au théorème de Torelli pour les variétés symplectiques irréductibles, C. R. Acad. Sci. Paris Sér. I Math. 299 (1984), no. 14, 681-684. MR770463 (87c:14039)

15. Classes de cohomologie positives dans les variétés kählériennes compacts [d'après Boucksom, Demailly, Nakayama, Păun, Peternell ...], Séminaire Bourbaki. Volume 2004/2005. Exposés 938-951. Paris: Société Mathématique de France. Astérisque 307, 199-228, Exp. No. 943 (2006), 2006. MR2296419 (2009a:32026)

16. M. Haiman, Hilbert schemes, polygraphs and the Macdonald positivity conjecture, J. Amer. Math. Soc. 14 (2001), no. 4, 941-1006. MR1839919(2002c:14008)

17. B. Hassett and Y. Tschinkel, Intersection numbers of extremal rays on holomorphic symplectic varieties, Asian J. Math. 14 (2010), 303-322. MR.2755719

18. D. Huybrechts, Compact hyper-Kähler manifolds: basic results, Invent. Math. 135 (1999), no. 1, 63-113. MR1664696 (2000a:32039)

19. , Erratum to: Compact hyperkähler manifolds: basic results, Invent. Math. 152 (2003), no. 1, 209-212. MR 1965365 (2004c:32041)

20. E. Markman, A survey of Torelli and monodromy results for holomorphic-symplectic varieties, Proc. of the Conference "Complex and Differential Geometry", Springer Proceedings in Mathematics, Volume 8, 2011, 257-322.

21. _ Integral constraints on the monodromy group of the hyperKähler resolution of a symmetric product of a K3 surface, Internat. J. Math. 21 (2010), no. 2, 169-223. MR2650367 (2011b:14008)

22. C. T. McMullen, Dynamics on K3 surfaces: Salem numbers and Siegel disks, J. Reine Angew. Math. 545 (2002), 201-233. MR.1896103(2003a:37057)

23. S. Mukai, Finite groups of automorphisms of K3 surfaces and the Mathieu group, Invent. Math. 94 (1988), no. 1, 183-221. MR958597 (90b:32053)

24. Y. Namikawa, Counter-example to global Torelli problem for irreducible symplectic manifolds, Math. Ann. 324 (2002), no. 4, 841-845. MR.1942252 (2003k:14008a)

25. V. V. Nikulin, Finite groups of automorphisms of Kählerian K3 surfaces, Trudy Moskov. Mat. Obshch. 38 (1979), 75-137. MR544937 (81e:32033)

26. K. G. O'Grady, Irreducible symplectic 4-folds and Eisenbud-Popescu-Walter sextics, Duke Math. J. 134 (2006), no. 1, 99-137. MR.2239344(2007e:14062)

27. K. Oguiso, Tits alternative in hyperkähler manifolds, Math. Res. Lett. 13 (2006), no. 2-3, 307-316. MR2231119 (2007e:14023) 
28. _ Automorphisms of hyperkähler manifolds in the view of topological entropy, Algebraic geometry, Contemp. Math., vol. 422, Amer. Math. Soc., Providence, RI, 2007, pp. 173-185. MR.2296437 (2008a:14055)

29. , Bimeromorphic automorphism groups of non-projective hyperkähler manifolds - a note inspired by C. T. McMullen, J. Differential Geom. 78 (2008), no. 1, 163-191. MR 2406267 (2009g:32037)

30. , A remark on dynamical degrees of automorphisms of hyperkähler manifolds, Manuscripta Math. 130 (2009), no. 1, 101-111. MR2533769 (2010m:32021)

31. K. Oguiso and S. Schröer, Enriques manifolds, J. Reine Angew. Math. (to appear) (2011).

32. H. Sterk, Finiteness results for algebraic K3 surfaces, Math. Z. 189 (1985), no. 4, 507-513. MR786280 (86j:14038)

33. M. Verbitsky, Cohomology of compact hyper-Kähler manifolds and its applications, Geom. Funct. Anal. 6 (1996), no. 4, 601-611. MR.1406664 (98a:53069)

Laboratoire J.A. Dieudonné UMR CNRS 6621, Université de Nice Sophia-Antipolis, Parc Valrose, F-06108 Nice, France

E-mail address: Samuel.Boissiere@unice.fr

URL: http://math.unice.fr/ sb/

Current address: Laboratoire de Mathématiques et Applications, UMR CNRS 6086, Université de Poitiers, Téléport 2, Boulevard Marie et Pierre Curie, F-86962 Futuroscope Chasseneuil, France

E-mail address: samuel.boissiere@math.univ-poitiers.fr

$U R L:$ http://www-math.sp2mi.univ-poitiers.fr/ sboissie/

Laboratoire de Mathématiques et Applications, UMR CNRS 6086, Université de Poitiers, Téléport 2, Boulevard Marie et Pierre Curie, F-86962 Futuroscope ChasSeneuil, France

E-mail address: sarti@math.univ-poitiers.fr

$U R L:$ http: //www-math.sp2mi.univ-poitiers.fr/ sarti/ 